\title{
Acute Fibrinous and Organising Pneumonia in Whipple's disease
}

\author{
P.A. Canessa1, L. Pratticò ${ }^{1}$, M. Sivori1, P. Magistrelli2, \\ F. Fedeli3, A. Cavazza4, G. Calcina²
}

ABSTRACT: Acute Fibrinous and Organising Pneumonia in Whipple's disease. P.A. Canessa, L. Pratticò M. Sivori, P. Magistrelli, F. Fedeli, A. Cavazza, G. Calcina.

We report a case of acute fibrinous and organising pneumonia in Whipple's disease with lung improvement after antibiotic therapy. In our knowledge this is the first report of Whipple's disease with acute fibrinous and organising pneumonia.

Monaldi Arch Chest Dis 2008; 69: 4, 186-188.

Keywords: Acute fibrinous and organising pneumonia, Whipple's disease, Acute lung injury, Diffuse alveolar damage.

1 UO Pneumologia - Ospedale S. Bartolomeo, Sarzana (SP);

2 UO Chirurgia generale - Ospedale S. Andrea, La Spezia;

3 Anatomia Patologica - Ospedale S. Andrea, La Spezia;

4 UO Anatomia Patologica-Arcispedale S. Maria Nuova, Reggio Emilia, Italy.

Correspondence: Pier Aldo Canessa, UO Pneumologia, Ospedale S Bartolomeo, Via Cisa sud, 19038, Sarzana (SP), Italy; e-mail:pieraldo.canessa@asl5.liguria.it

\section{Introduction}

Acute fibrinous and organising pneumonia (AFOP) is a histological pattern associated with acute lung injury and is different from diffuse alveolar damage (DAD); it has been reported in a variety of clinical conditions and/or associated with various substances and environments, but not in Whipple's disease.

We report a case of AFOP in Whipple's disease.

\section{Case Report}

In October 2006, a 60 year-old woman was admitted with alveolar consolidation in the lower left lobe and mild bilateral pleural effusion. Three months prior to admission she had complained of diarrhoea, progressive dyspnoea and dry cough, and reported a weight loss of $10 \mathrm{~kg}$. The patient had never smoked or taken drugs and had always been a housewife. A duodenal biopsy revealed periodic acid Schiff (PAS) positive macrophages in the lamina propria (fig. 1), and by late September, Whipple's disease was considered to be likely. A computed tomography of the chest showed pulmonary infiltration with air bronchogram in the lower left lobe and mild bilateral pleural effusion (fig. 2A). Infectious agents (in blood: Mycoplasma IGG and IGM, Chlamidya IGG, CMV-DNA and CMV-antigen, HIV, Aspergillus-antigen; in urine: Pneumococcus and Legionella; in pleural effusion: Gram stain and special stains for acid-fast bacilli and fungi, TB-DNA, routine cultures for bacteria, fungi and mycobacteria) were not detectable. A thoracoscopic biopsy of the lower-left lobe showed acute parenchymal damage with endoalveolar fibrin "balls", some aspecific foamy macrophages and type 2 pneumocyte hyperplasia (fig. 3); no hyaline membranes were present. A pleural biopsy showed non-specific pleuritis. The histological features were consistent with AFOP and non-specific pleuritis.

Antibiotic therapy was commenced with trimethoprim and sulphamethoxazole orally twice daily for three months, which was followed by doxycycline $100 \mathrm{mg} /$ day. Five months after beginning therapy, diarrhoea and respiratory symptoms completely disappeared and the general condition of the patient improved. CT scans of the chest revealed the absence of lower-left lobe consolidation and a reduction of pleural effusion (Fig. 2B). This, together with the patient's response to antibiotics, led us to diagnose Whipple's disease with AFOP.

\section{Discussion}

The term AFOP was first used by Beasley and colleagues in 2002 [1]. They described the presence of intra-alveolar fibrin in the form of fibrin 'balls' and organising pneumonia with patchy distribution as the main histological features of this type of lung injury. Similar to other patterns of acute lung injury, the AFOP pattern has been reported in a variety of clinical conditions and/or as- 


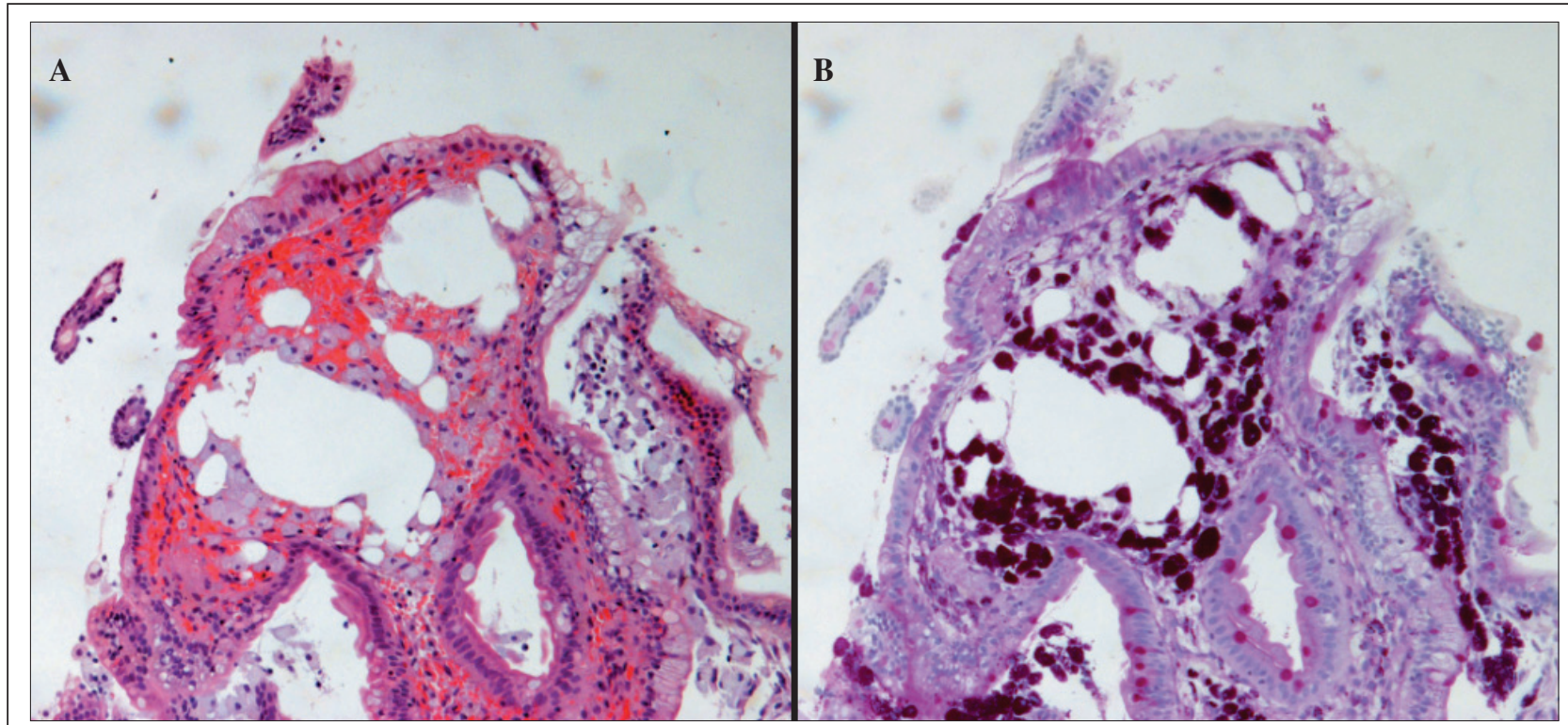

Fig. 1. - Duodenal biopsy: the villus is distented and shows foamy macrophages and dilated lacteals with large lipid droplets. (A. Hematoxylin-Eosin 100x, B. PAS 100x).

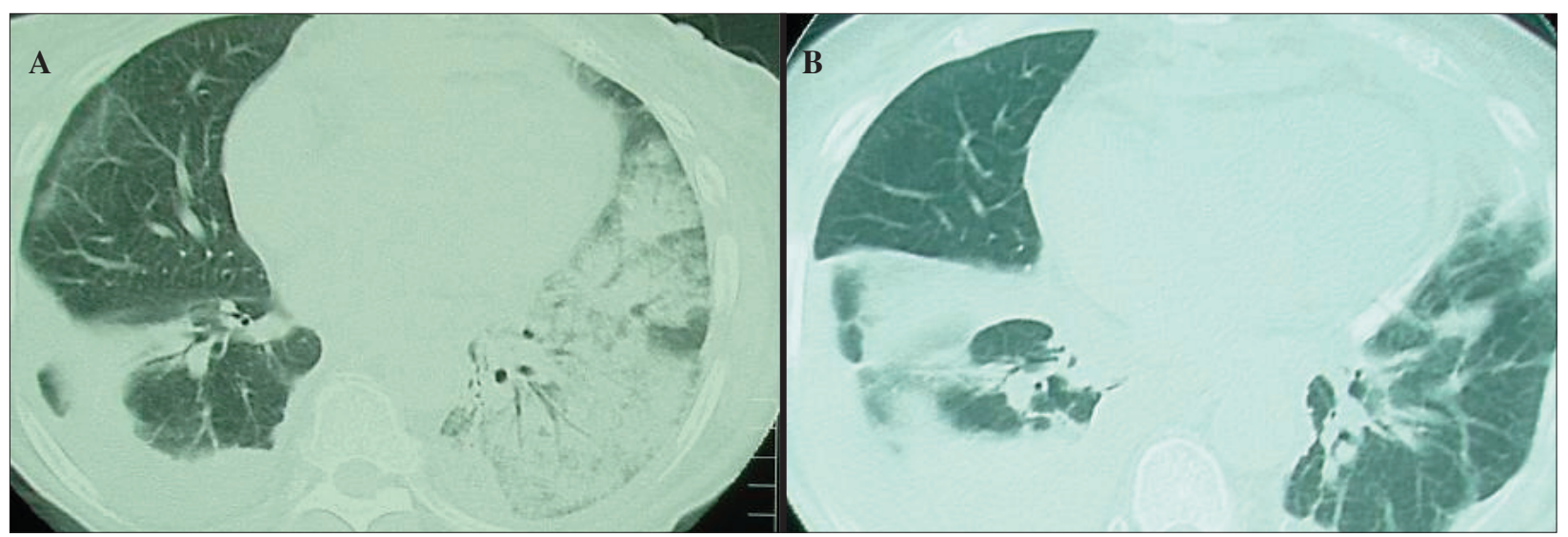

Fig. 2. - CT scan.

A. Alveolar consolidation in left lower lobe and small bilateral pleural effusion. B. Absence of lower-left lobe consolidation after five months.

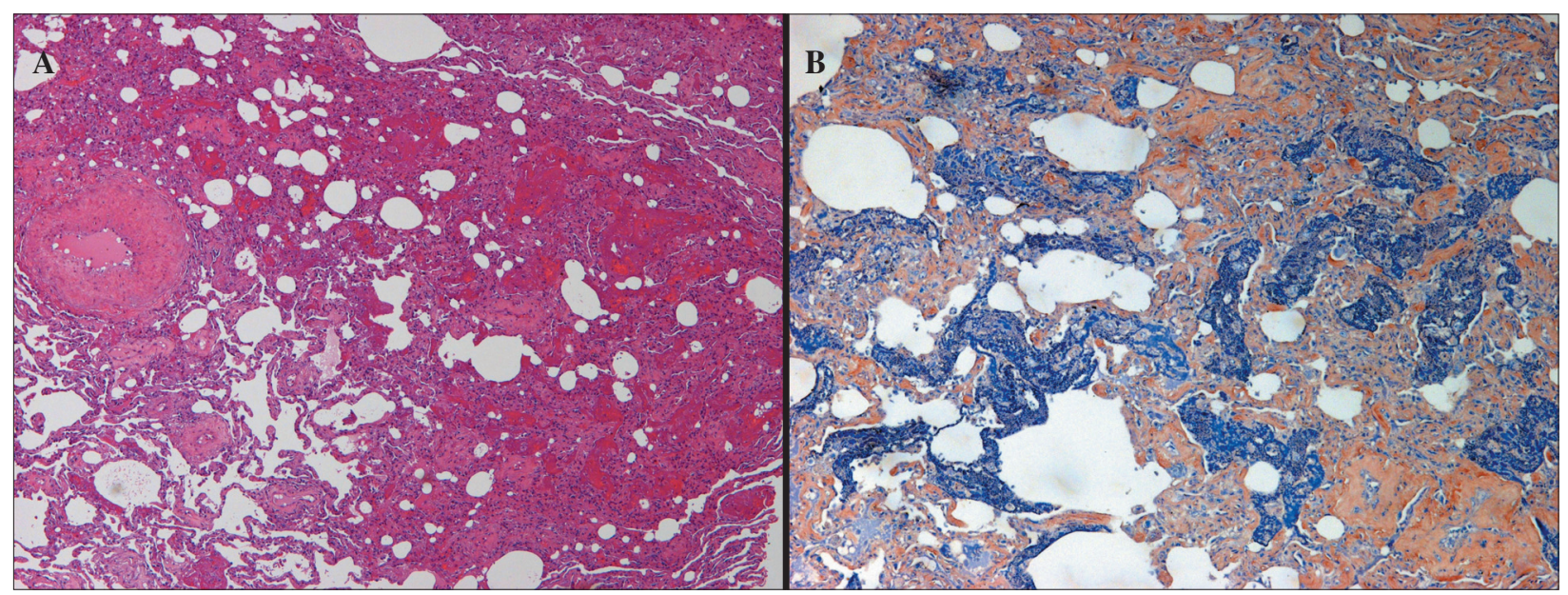

Fig. 3. - Lung biopsy:

A. Endoalveolar accumulation of fibrin balls, with scattered nuclear debris. No hyaline membranes are present (Hematoxylin-Eosin, 200x).

B. Fibrin balls stained with PTAH (200x).

sociated with various substances and environments: collagen vascular disease, amiodarone toxicity, infectious pneumonia (Haemophilus influen$z a$, Acinetobacter $s p$.), lymphoma, hairspray, con- struction work, coal mining, and zoological work $[1,2]$; it is often idiopathic, as the condition frequently appears without any identifiable cause or association [1]. 
Whipple's disease is a rare chronic infectious disorder first described in 1907 by G.H. Whipple. In addition to the small intestine, Whipple's disease can involve the remainder of the gastrointestinal tract, as well as the lymph nodes, joints, nervous system, heart, eyes, haematopoietic system, liver, and other organs. Pleuropulmonary involvement is reported in $13-14 \%$ [3, 4] of cases: usually pleural effusion and/or pulmonary hypertension [5].

Whipple's disease is caused by the recently identified bacterium Tropheryma Whipplei and there is evidence that altered immune function plays a role in the manifestation of the disease.

This is the first diagnosed case of duodenal Whipple's disease with AFOP. Tropheryma Whipplei causes an infectious systemic disease and we suggest that it could also cause acute lung injury with an AFOP histological pattern.

\section{References}

1. Beasley MB, Franks TJ, Galvin JR, Gochuico B, Travis WD. Acute Fibrinous and Organising Pneumonia - A Histologic Pattern of Lung Injury and Possible Variant of Diffuse Alveolar Damage. Arch Pathol Lab Med 2002; 126: 1064-70.

2. Balduin R, Giacometti C, Saccarola L, et al. Acute fibrinous and organising pneumonia in a patient with collagen vascular disease "stigma". Sarcoidosis Vasc Diffuse Lung Dis 2007; 24: 78-80.

3). Fenollar F, Puéchal X, Raoult D. Whipple's disease. $N$ Engl J Med 2007; 356: 55-66.

4. Durand DV, Lecomte C, Cathebras P, Rousset H, Godeau P. Whipple's disease. Clinical review of 52 cases. The SNFMI Research Group on Whipple's disease. Societe Nationale Francaise de Medecine Interne. Medicine (Baltimore) 1997; 76: 170-84.

5. Riemer H, Hainz R, Stain C, et al. Severe pulmonary hypertension reversed by antibiotics in a patient with Whipple's disease. Thorax 1997; 52: 1014-15.

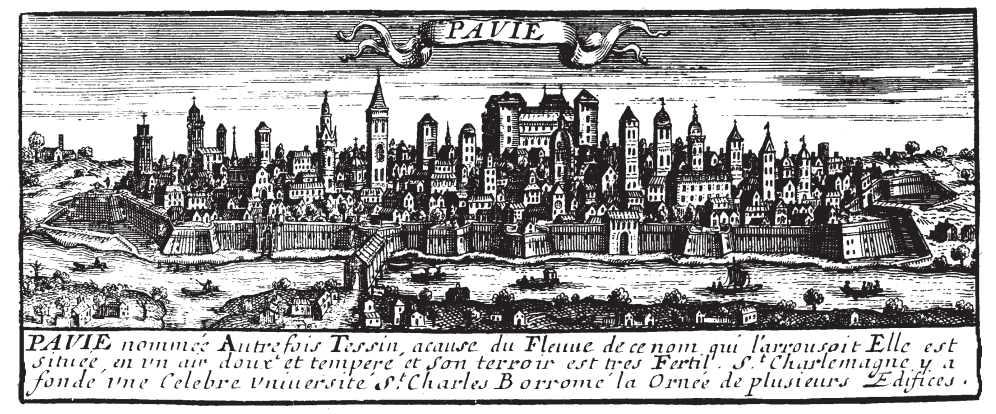

